\title{
Excitons and Peierls Distortion in Conjugated Carbon Nanotubes
}

\author{
Sergei Tretiak, ${ }^{*}, \uparrow$ Svetlana Kilina, ${ }^{, \neq}$Andrei Piryatinski, ${ }^{\dagger}$ Avadh Saxena, ${ }^{\dagger}$ \\ Richard L. Martin, ${ }^{\dagger}$ and Alan R. Bishop ${ }^{\dagger}$
}

Theoretical Division, Center for Nonlinear Studies (CNLS), and Center for

Integrated Nanotechnologies (CINT), Los Alamos National Laboratory,

Los Alamos, New Mexico 87545, and Department of Chemistry,

University of Washington, Seattle, Washington 98195-1700

Received September 18, 2006; Revised Manuscript Received November 12, 2006

\begin{abstract}
We investigate coupled excitonic and vibrational effects in carbon nanotubes using a time-dependent Hartree-Fock approach. The results reveal intricate details of excited-state dynamics. In the ground state, spontaneous uneven distribution of the $\pi$ electrons over the bonds (i.e., Peierls dimerization) is observed throughout the entire nanotube, particularly in large-radius CNTs. However, we demonstrate that vibrational relaxation following photoexcitations leads to substantial local distortion of the tube surface, overriding the Peierls dimerization. This mutually affects the electronic system, resulting in localized states (self-trapped excitons). These phenomena critically control photoinduced dynamics and charge transport in nanotube materials.
\end{abstract}

Single-walled carbon nanotubes (CNTs) are quasi-onedimensional structures composed of a one-atom-thick layer of graphite rolled into a tube. ${ }^{1}$ Their extended backbones support a delocalized mobile $\pi$-electron system. This results in a semiconductor or metal-like electronic structure with unique physical properties, promising for a number of technological applications. ${ }^{2-7}$ Electronic structure of CNTs based on tight-binding models predicts equally spaced subbands of valence and conduction bands with diverging density of states at band edges known as van Hove singularities. ${ }^{1}$ Recent experimental ${ }^{8-10}$ and theoretical ${ }^{11-16}$ studies have revealed the importance of electron-electron correlations caused by enhancement of Coulomb interactions due to one-dimensional confinement conditions. For example, the response of bound electron-hole pairs (excitons) is manifested in fluorescence ${ }^{8,17}$ and photoinduced optical absorption $^{9,10}$ spectra of CNTs. In addition to probing excitonic phenomena, recent research has shown that electronphonon coupling (vibrational effects) are important in carbon nanotubes as well. ${ }^{18-25}$ Strong phonon-assisted bands identified in single CNT photoluminescence spectra, ${ }^{19,20}$ and coherent phonon generation ${ }^{22}$ detected in time-resolved spectra, prove a substantial electron-phonon coupling.

An interplay of strong Coulomb correlations, electronphonon coupling, and elastic properties are typical features of all quasi-one-dimensional materials such as conjugated

\footnotetext{
* Corresponding author. E-mail: serg@lanl.gov.

tos Alamos National Laboratory.

$\doteqdot$ University of Washington.
}

organic $^{26,27}$ and organometallic ${ }^{28}$ polymers, and mix-valence chains, ${ }^{29}$ frequently giving rise to spontaneous symmetry breaking (Peierls distortion). This leads to rich physical phenomena such as metal-semiconductor transitions, solitons, polarons, breathers, and enables technological applications. Their exploration in the context of CNTs is complicated due to sample quality issues (experiment) and significant numerical effort involved in quantum-chemical studies (theory). Most of the theoretical investigations of CNTs have been carried out using model calculations or empirical tightbinding approximations, ${ }^{1,13,14}$ except several first-principle studies focused on a few snapshots of the electronic structure of narrow-diameter CNTs. ${ }^{15,16}$ Modeling of excited-state geometries and excited-state molecular dynamics, requiring multiple-step electronic structure calculations, have not been pursued due to numerical expense, except for a few model approaches. ${ }^{23}$ Thus, an understanding of coupled excitonic and vibrational interactions as a function of CNT diameter (curvature) and chirality is not yet available.

In the present study, we focus on the CNT photoinduced dynamics and the effect of spontaneous symmetry breaking, also known as Peierls distortion. The latter phenomenon is attributed to the instability of the Fermi energy states in quasi-one-dimensional metals. In the half-filled zone materials, the instability is removed by the electron-phonon interactions, leading to the gap opening at the Fermi level (metal-semiconductor transition) and induced by the folding of the Brillouin zone and related doubling of the real-space lattice period. ${ }^{30}$ Conjugated polymers provide an example 


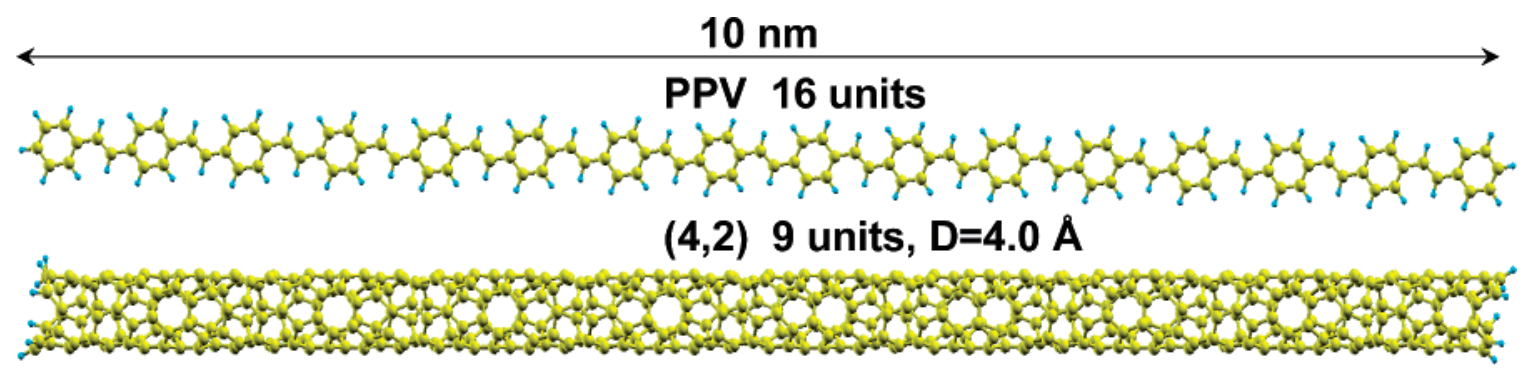

$(6,2) 7$ units, $D=5.7 \AA$

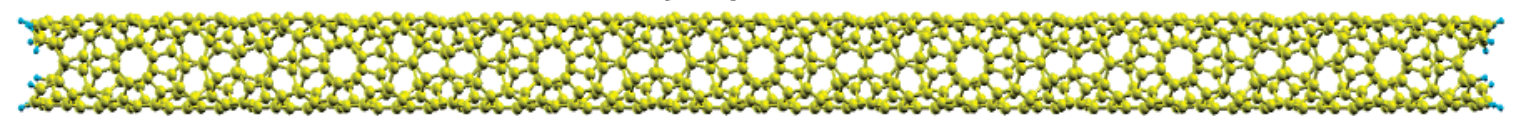

\section{$(7,6) 2$ units, $D=9.0 \AA$}

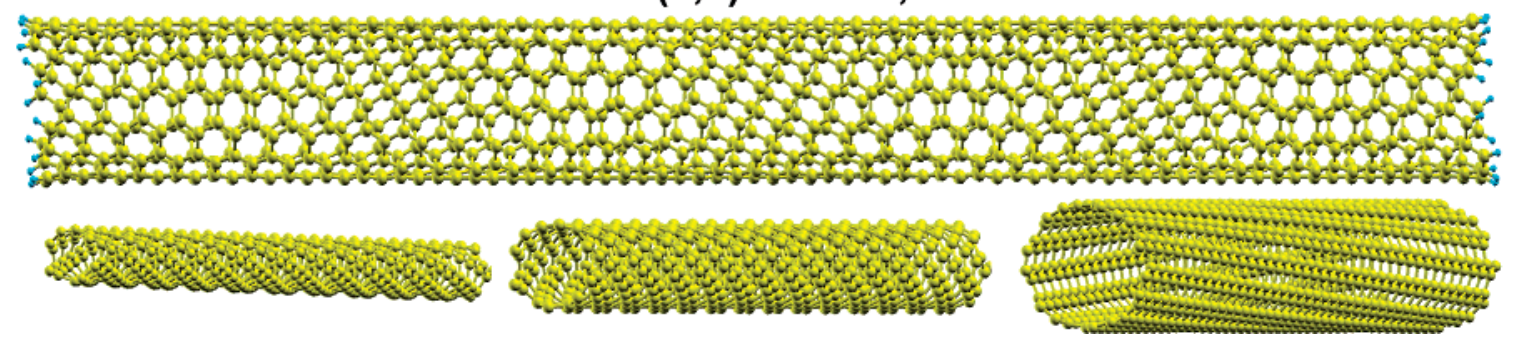

Figure 1. Molecular structure of poly phenylene-vinylene (PPV), and (4,2), (6,2), (7,6) CNTs with increasing diameter. All systems are finite, have the same length, and are terminated by hydrogen atoms to saturate chemical bonds. Lower panel represents a perspective depth view of the tubes for a visual comparison of their diameters and chiralities.

of such a transition with the gap opening of about $2 \mathrm{eV} .{ }^{26}$ The Peierls distortion has been extensively studied in metallic CNTs. ${ }^{1,31-35}$ In contrast to polymers, the produced band gap in the latter is much smaller than the energy of the thermal fluctuations. In the present study, we consider semiconductor CNTs and find that their surfaces experience the corrugation fully analogous to the lattice period doubling. Because this spontaneous symmetry breaking is associated with the electron-phonon interaction, we refer to it as Peierls distortion. However, this phenomenon cannot be considered as metal-semiconductor transition.

We use our recently developed excited state molecular dynamics (ESMD) approach ${ }^{36}$ to study photoinduced excitonic states of CNTs. This method has worked well in our recent studies for a number of organic chromophores, including conjugated polymers, providing quantitative accuracy and physical insight. ${ }^{27,36}$ Compared to model calculations, using our technique we expect to understand the interplay of $\pi$ and $\sigma$ bonding, curvature, and chirality effects in the excited states of CNTs. We focus on three semiconductor chiral tubes shown in Figure 1. Other CNT chiralities, which we have studied, exhibit similar trends described below. For comparison purposes, we also include a poly $(p-$ phenylene vinylene) (PPV) oligomer. All considered molecular systems have a finite length of $10 \mathrm{~nm}$ and comprise several repeat units. The finite size one-dimensional systems, when their lengths are significantly larger than the characteristic exciton sizes, are expected to reproduce well the properties in the infinite-size limit. For example, we estimate the differences between calculated finite tube excitation energies (Table 1) and their correspondent extrapolated values to be less than $0.05 \mathrm{eV}$. Calculations of conjugated
Table 1. Diameters and Calculated Spectroscopic Observables (Excitation Energies, Stokes Shifts, and Excited-State Vibrational Relaxations) of $\mathrm{CNTs}^{a}$

\begin{tabular}{cccccc}
\hline $\begin{array}{c}\text { polymer } \\
\text { or tube }\end{array}$ & $\begin{array}{c}D \\
(\AA)\end{array}$ & $\begin{array}{c}\mathrm{EX} 1 \\
(\mathrm{eV})\end{array}$ & $\begin{array}{c}\mathrm{EX} 2 \\
(\mathrm{eV})\end{array}$ & $\begin{array}{c}\text { Stokes } \\
\text { shift } \\
(\mathrm{meV})\end{array}$ & $\begin{array}{c}\text { vibrational } \\
\text { relaxation } \\
(\mathrm{meV})\end{array}$ \\
\hline PPV & & $2.54(2.4)$ & & 120 & 56 \\
$(4,2)$ & 4.0 & 1.57 & 2.92 & 2.4 & 1.3 \\
$(6,2)$ & 5.7 & $1.24(1.39)$ & $2.63(2.96)$ & 9.6 & 4.7 \\
$(7,6)$ & 9.0 & $0.98(1.11)$ & $2.06(1.92)$ & 52 & 32
\end{tabular}

${ }^{a}$ Available experimental excitation energies ${ }^{8}$ are given in parentheses.

oligomers provide a familiar reference example of such an approach. ${ }^{27,36}$ Optimal geometries of the ground state, the first optically allowed excited state, and the ground-state vibrational normal modes have been obtained for all four molecules, as described in ref 22 and outlined in the Methods section. Ground- and excited-state geometries provide input structures for calculating optical absorption and emission spectra, respectively.

To study the exciton structure, we calculate up to the 200 lowest excited states for each CNT from Figure 1 at the ground-state geometry. The computed states are strongly delocalized $\pi-\pi$ excitations, which are optically accessible and span most of the first (EX1) and second (EX2) excitonic bands. The lowest state in each excitonic band collects nearly all of the oscillator strength from its parent band. This results in characteristically strong EX1 and EX2 peaks in the CNT's linear absorption spectra probed by a variety of spectroscopic techniques. ${ }^{9,10,19-22}$ In contrast, only the lowest-energy (band gap) transition in PPV has strong oscillator strength. This state is also responsible for the polymer's fluorescence. ${ }^{36}$ The 
situation may be different in CNTs because there are several optically forbidden exciton states, nearly isoenergetic to the allowed EX1 state. The relative ordering of "bright" and "dark" states may be responsible for the poor fluorescence efficiency of the CNTs. ${ }^{13}$ We found that this ordering is highly method-dependent and obscured by vibrational relaxation (to be published as a separate contribution). Here we focus on the EX1 properties at the excited-state geometry related to the nanotube emission.

A comparison of ground- and excited-state geometries of CNTs and PPV is illustrated in Figure 2. An important feature of a conjugated polymer geometry is the bond length alternation $^{26,27}$ due to an uneven distribution of the $\pi$ electrons over the bonds (Peierls distortion). Figure 2A shows the lengths of carbon-carbon bonds along the chain, where periodic variation of single (long) and double (short) bonds is evident. This bond-length alternation is reduced over the exciton spatial region in the excited-state geometry, indicating a fundamental exciton self-trapping phenomenon. ${ }^{36}$ To analyze nanotube geometries, we inspect deviations of the tube surface from the perfect cylinder. In the smallest $(4,2)$ tube, these deviations (not shown) are significant because of considerable bonding system strain due to rolling of graphite into a cylinder with a diameter comparable to the phenyl ring size. As expected, a larger $(6,2)$ tube has more cylindrical geometry (not shown). The most interesting case is our widest $(7,6)$ tube. Tubes of similar diameter are very common in the experimental samples. The tube surface is corrugated in the ground-state geometry. Figure 2B shows that the radial distance of carbon atoms periodically changes along the tube axis (the labeling is shown in Figure 2C, left panel). The "belts" of "red" and "blue" carbon atoms are buckled inside and outside the tube, respectively. This obviously makes "red" bonds shorter and "blue" bonds longer, a property which is further enhanced by the corresponding rearrangement of bonds on the tube surface (Figure 2C). This effect is caused by the interactions within the delocalized $\pi$-electron system, rather than by changes of $\sigma$-bonds due to curvature. In fact, it is a manifestation of the Peierls dimerization previously explored in the CNT ground state context ${ }^{1,31}$ analogous to that commonly observed in conjugated polymers (compare Figure 2A and B). Because of its more rigid structure, the respective nanotube bondlength alternation is about $\sim 0.02 \AA$ vs $\sim 0.1 \AA$ in PPV. The similarity of excited-state vibrational relaxation is even more striking: The nanotube surface becomes locally less distorted, corresponding to reduction of the bond-length alternation (Figure $2 \mathrm{C}-\mathrm{D}$ ). Even though the geometrical changes are small $(\sim 0.002-0.02 \AA)$, this effect still leads to the measurable vibrational progression in the emission spectra (Figure 2E). ${ }^{24}$

These delicate geometric features have profound effects on the CNT electronic structure. Table 1 summarizes our main computational results. Overall, the calculated excitation energies agree well (within $0.2 \mathrm{eV}$ ) with available experimental data. ${ }^{8}$ Such accuracy is typical for semiempirical molecular calculations..$^{27,36}$ The relevant excitonic states of all molecules are analyzed in Figure 3. The contour plots

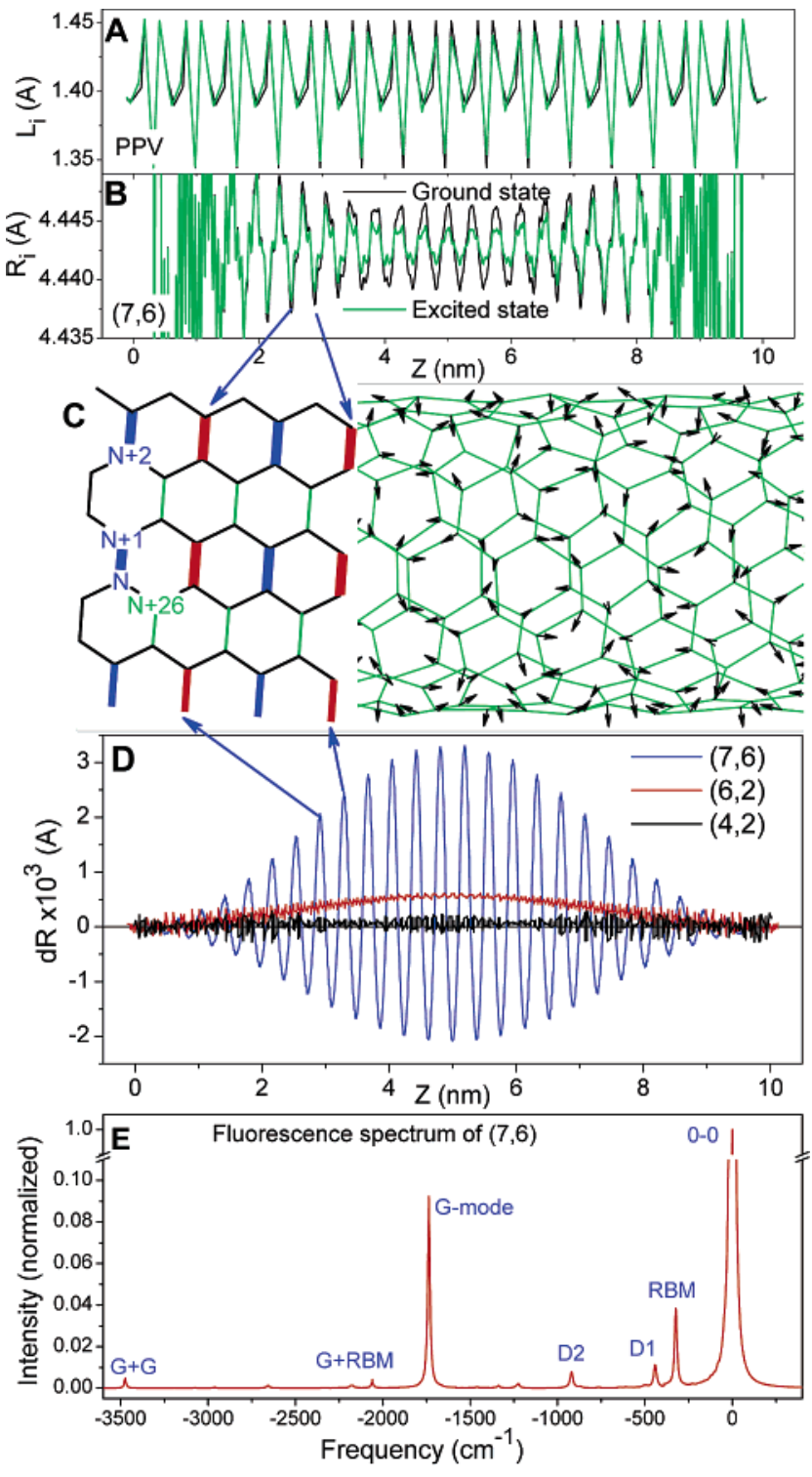

Figure 2. Comparison of calculated-excited (EX1) and groundstate optimal geometries. (A) Variation of carbon-carbon bond length along the PPV chain. (B) Right panel: Variation of carbon radial distance from the center along the $(7,6)$ tube axis. (C) Directions of nuclear motion induced by vibronic relaxation on the excited state. Stretching (G-mode) and radial breathing (RBM) motions are clearly visible along arrow directions. The left panel in (B) shows the corresponding hexagonal lattice and atoms buckling inside (red) and outside (blue) of the nanotube surface due to a Peierls distortion. (D) Change of the radius $d R$ with carbon coordinate along the tube $Z$ axis showing corrugation of the tube surface in the excited state. (E) Calculated fluorescence spectrum showing the vibrational replica of normal modes coupled to the electronic degrees of freedom.

(first column in Figure 3) represent the transition density matrices between the ground and electronically excited states, as "topographic" maps that reflect the single electron reduced density matrix changes upon molecular photoexcitation. ${ }^{27}$ The matrix diagonal and off-diagonal sizes characterize the distribution of an excitonic wave function over the chain, namely center-of-mass $\left(L_{\mathrm{D}}\right)$ and distance between particles $\left(L_{C}\right)$, respectively. To establish a reference point, we start with the polymer (PPV) chain. The results are shown in the 

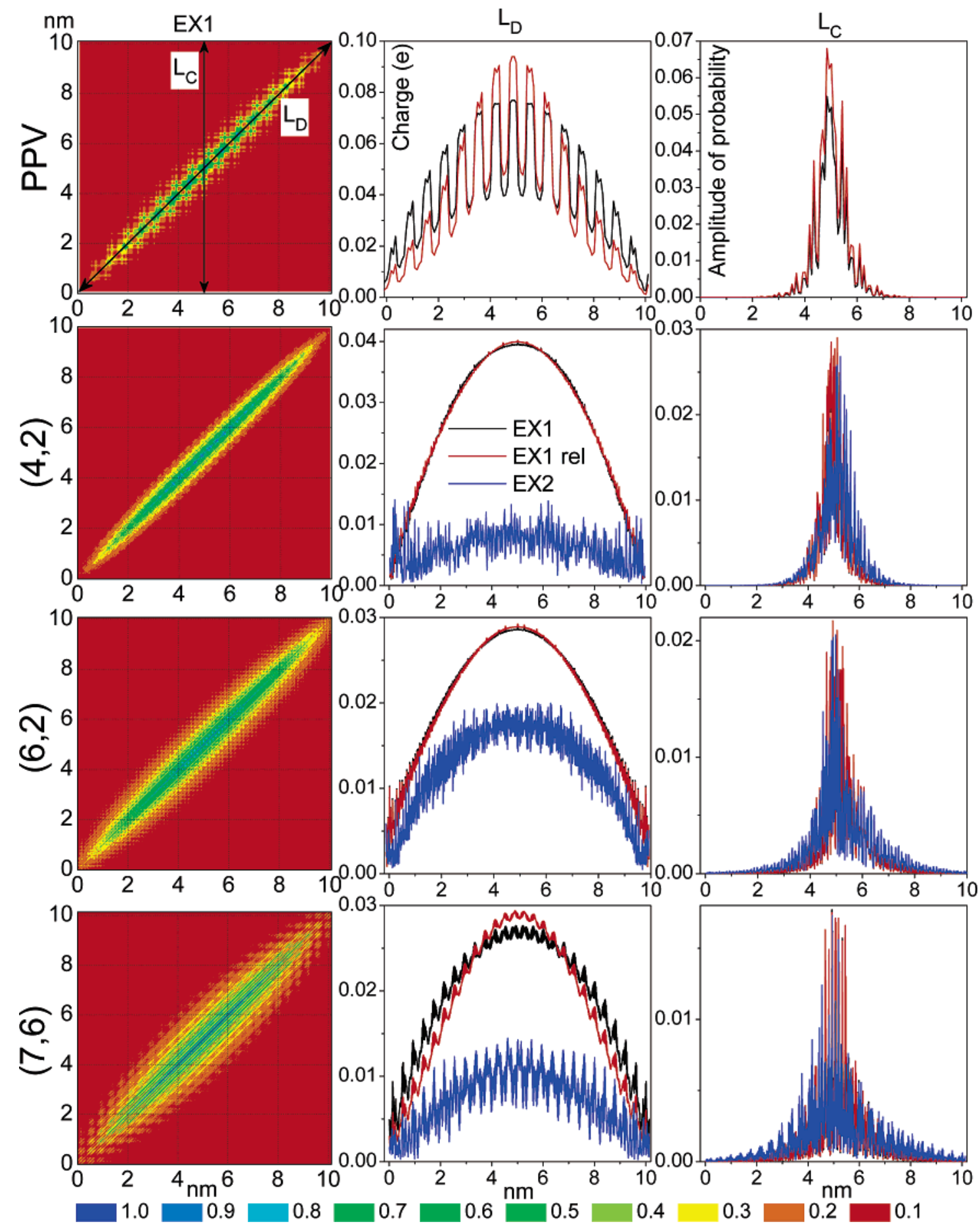

Figure 3. Analysis of transition density matrices from the ground state to optically active excited states of the molecules shown in Figure 1. Two-dimensional contour plots of matrices corresponding to the band gap transition are shown in the first column. Each plot depicts probabilities of an electron moving from one molecular position (horizontal axis) to another (vertical axis) upon electronic excitation. The color code is shown in the bottom line. The second (third) column represents diagonal (off-diagonal) slices of matrices along the $L_{\mathrm{D}}\left(L_{\mathrm{C}}\right)$ direction, showing delocalization patterns of excited states.

first row. The contour plot illustrates that the center of mass of a photoexcited electron-hole pair is delocalized over the entire chain (diagonal in the plot). The exciton size (maximal distance between electron and hole) is about $2 \mathrm{~nm}$ (largest off-diagonal extent of the nonzero matrix area). The relevant $L_{\mathrm{D}}$ and $L_{\mathrm{C}}$ cross-sections are shown in the second and third columns in Figure 3, respectively. The $L_{\mathrm{D}}$ slice (black curve) for PPV has distinct peaks associated with the strong change of the electronic density on the double bonds of vinyl groups upon photoexcitation (namely, a reduction of Peierls dimerization). The electronic excitation then relaxes along the excited-state potential surface and ultimately decays radiatively back to the ground state (fluorescence). Because of the coupling to vibrational degrees of freedom (electronphonon coupling), an exciton distorts the lattice in the middle of the chain (Figure 2A) and localizes itself in this region 
(compare the red and black curves on the $L_{\mathrm{D}}$ slice), which is typical for conjugated polymers. ${ }^{26,36}$ The off-diagonal exciton size $L_{C}$ is not affected by vibrational relaxation.

The second row in Figure 3 characterizes excitonic properties of a $(4,2)$ tube. The EX1 of this tube looks very similar to the band gap exciton of polymer with the same $L_{\mathrm{D}}$ and $L_{\mathrm{C}}$ sizes. However, the vibrational relaxation of the EX1 state (Table 1) is negligible (the black and red curves on the $L_{\mathrm{D}}$ and $L_{\mathrm{C}}$ slices coincide). This lack of vibrational relaxation in narrow CNTs is attributed to the strong strain in the $\sigma$-bonding network and weaker (compared to the planar graphite) interactions within the $\pi$-electron system due to large curvature. Compared to EX1, a higher energy photoexcitation EX2 is uniformly delocalized along the CNT with smaller changes of the $\pi$-electron density (blue curve on the $L_{\mathrm{D}}$ slice). This excitation has the same off-diagonal size as EX1 and is sensitive to the CNT chirality, showing up as an asymmetric shape in the $L_{\mathrm{C}}$ plot. Compared to the $(4,2)$ tube, the $(6,2)$ CNT has a smaller curvature and, consequently, a better $\pi$-electron conjugation and mobility. This results in a larger (about $4 \mathrm{~nm}$ ) exciton size. The curvature-induced strain of the $\sigma$-bonding network is still strong, so the EX1 vibrational relaxation in $(6,2)$ is relatively weak (Table 1) and appears as a slight bulge in the center of the tube (Figure 2D). EX2 in this tube has a pronounced asymmetry, as evidenced by the $L_{\mathrm{C}}$ plot.

The bottom row in Figure 3 summarizes the excitonic properties of the $(7,6) \mathrm{CNT}$. Both EX1 and EX2 in this tube have sizes of about $6 \mathrm{~nm}$, which is still smaller than the 10 $\mathrm{nm}$ tube length, minimizing edge effects. Compared to the narrower tubes, the $L_{\mathrm{D}}$ plots for all excited states have periodic variation of the changes in the electronic density due to photoexcitation, which is very similar to that observed on the $L_{\mathrm{D}}$ slice for the conjugated polymer, signaling the effect of the Peierls distortion on the electronic degrees of freedom. Moreover, distortion of the nanotube surface at the center (Figure 2D) in the excited state evidence exciton selftrapping by vibrational relaxation, which is well pronounced in the electronic degrees of freedom as well (red and black curves on the $L_{\mathrm{D}}$ slice). Compared to PPV, where the diagonal exciton localization size is about $5 \mathrm{~nm}$, this length in the $(7,6)$ tube is about $8-9 \mathrm{~nm}$. Excited-state vibrational relaxation ("exciton cooling") and the associated Stokes shift are substantial, making it possible to probe this phenomenon spectroscopically, such as routinely done in other materials. ${ }^{26,29}$ It is interesting to point that bound dynamical exciton-phonon states were observed in the previous theoretical and experimental studies, ${ }^{23,24}$ which confirms strong electron-vibrational interplay in carbon nanotube materials. The impact of chemical defects, solvent stabilization, and intertube interactions on this dynamics is a subject of future studies.

Finally, we discuss typical vibrational motions strongly coupled to the electronic degrees of freedom. Comparison of the ground- and excited-state geometries in the $(7,6) \mathrm{CNT}$ (Figure 2C and D) shows that excited-state relaxation rearranges bond-length alternation and slightly bulges the tube. This suggests a coupling to the longitudinal G and radial breathing (RBM) vibrational modes, which is the largest among all vibrational coordinates. Ground- and excited-state geometries and vibrational normal modes make it possible to calculate the vibronic structure of photoluminescent spectra, as outlined in the Methods section. Figure 2E shows the computed spectrum for $(7,6) \mathrm{CNT}$. Vibrational replicas of the G-mode and the RBM are clearly seen. The intensity of the G-mode $0-1$ transition is about $9 \%$ of the $0-0$ line, which roughly corresponds to the value 0.1 of the related Huang-Rhys factor. This concurs with experimental emission and Raman spectra..$^{8,17,19-21,37}$ In particular, it agrees well with experimentally measured spectral weight transfer from the exciton to the exciton- phonon sideband. ${ }^{24}$ Furthermore, the small overtones $\mathrm{G}+\mathrm{G}$ and $\mathrm{G}+\mathrm{RBM}$, which can be identified in Figure 2E, are also observed in experimental 2D absorption/emission plots. ${ }^{19,20}$

In conclusion, we have investigated excited-state properties of carbon nanotubes using reliable semiempirical quantumchemical techniques. Calculated typical exciton-phonon couplings correspond to the Huang-Rhys factors of about 0.03 and 0.1 for RBM and G-mode $((7,6)$ tube), respectively. ${ }^{38}$ This places carbon nanotubes in a regime of a weak exciton-phonon coupling strength compared to many molecular materials such as conjugated polymers, where the Huang-Rhys parameters are close to one. ${ }^{36}$ In contrast, the CNT vibrational couplings are far exceeding those observed in typical solid-state materials, and are substantial for material's photophysical properties. Significant electronic correlation effects and electron-phonon coupling are found to dominate photoinduced processes, which is similar to other quasi-1D electronic systems. ${ }^{26,27,29}$ Vibrational relaxation in the excited state leads to exciton self-trapping on about 8 $\mathrm{nm}$ length scale, demonstrating that, in terms of "energy minimization", it is more preferable to form a local lattice distortion (self-trapped exciton) rather than a uniform bond alternation (Peierls dimerization) observed in the ground state. Inhomogeneous dielectric environment (solvent and intertube interactions) should facilitate this localization even further. The photoinduced dynamics in CNT materials may also be strongly affected by "dark" excitonic states, ${ }^{13}$ which have about the same vibrational coupling and reorganization energies and nearly isoenergetic to the allowed "bright" EX1 state. These effects intensify with increase of the tube diameter, reflecting enhanced mobility of $\pi$ electronic systems. However, their subsequent reduction is expected due to smaller $\pi-\sigma$ mixing and vanishing Peierls dimerization in superwide CNTs. ${ }^{1}$ Even stronger vibrational and carrier trapping effects are expected in the charge transport. Observed interplay between coupled electronic and vibrational degrees of freedom and curvature directly influences the photophysical properties of carbon nanotube materials and needs to be accounted for when designing specific lightdriven nanotechnological applications based on CNTs.

The Austin model 1 (AM1) Hamiltonian ${ }^{39}$ and the ESMD computational package ${ }^{36}$ were used for all calculations presented in this letter. The ESMD approach calculates the excited-state potential energy as $E_{\mathrm{e}}(q)=E_{\mathrm{g}}(q)+\Omega(q)$ in the space of nuclear coordinates $q$, which span the entire 
$(3 N-6)$ dimensional space, $N$ being the total number of atoms in the molecule. Here $\Omega(q)$ is an electronic transition frequency of the photoexcited molecule. The program uses analytic derivatives of $E_{\mathrm{e}}(q)$ with respect to each nuclear coordinate $q_{i}$ to calculate forces on the fly and subsequently to step along the excited-state hypersurface using these gradients. A standard Verlet molecular dynamics algorithm has been used for propagation of the Newtonian equations of motion. All computations start from the optimal molecular geometry, where the potential energy $E_{\mathrm{g}}(q)$ is minimal. If no dissipative processes are included, the total molecular energy $E_{\mathrm{e}}(q)$ is conserved. Inclusion of an artificial dissipative force into the ESMD equations of motion allows determination of the minimum of the excited-state potential energy surface $E_{e}(q)$, corresponding to the relaxed geometry.

Unsaturated chemical bonds in the initial nanotube geometries shown at the CNT ends have been capped with hydrogen atoms to remove a midgap state caused by dangling bonds (Figure 1). Ground-state optimal geometries and respective sets of vibrational normal modes of all molecules (Figure 1) are calculated using the MOPAC 2002 package. ${ }^{40}$ Alternatively, geometry optimizations are conducted in the infinite molecules by imposing one-dimensional periodic boundary conditions. Both approaches result in the nearly identical optimal geometries in the bulk of the tube (1-2 $\mathrm{nm}$ away from the tube ends). Subsequently discussed Peierls distortion effects are not artifacts introduced by the tube ends. ${ }^{41}$ The ESMD approach was next applied to optimize the geometry of the first optically allowed excited state in the space of all vibrational coordinates $q$. We note that excited-state geometry optimization rearrange carbon atoms in the middle of the tube (see Figure 2D), consequently, the tube ends are not affecting the excited-state dynamics as well.

Optimized ground- and excited-state geometries are further used to calculate excited-state electronic structures, which are related to the absorption and emission processes, respectively. These calculations characterize each excitedstate via the vertical excitation energy, oscillator strength, and electronic transition density matrix (or electronic normal mode). The latter bears important information on the changes in the molecular density and coherences induced upon optical excitation. This can be visualized using a two-dimensional real-space representation (Figure 3). The diagonal elements $\left(\xi_{v}\right)_{n n}$ of the matrices describe the net charge induced at the $n$th atom by the external field. The off-diagonal elements $\left(\xi_{v}\right)_{m n}$ with $m \neq n$ represent the joint probability amplitude of finding an electron and a hole located at the $m$ th and $n$th atoms, respectively. ${ }^{27}$ Thus, the transition density matrices show the spatial extent of electronic transitions with optically induced charges and electronic coherences.

The ground- and excited-state optimal geometries and vibrational normal modes of the ground state were further used to calculate line-shapes of fluorescence spectra of the $(7,6)$ CNT shown in Figure 2E. This can be readily achieved within the Condon approximation for displaced multidimensional harmonic oscillators. The vibrational overlap integrals $\left|\left\langle 0 \mid v_{n}\right\rangle\right|^{2}=e^{-S} S_{n}^{\nu} / v$ ! (Franck-Condon factors), govern the probability of emission from transition between the vibra- tional level 0 in the lowest excited state and a vibrational level $n$ in the ground state. These quantities, in turn, depend on the dimensionless displacements $\Delta_{n}$ of each normal mode with Huang-Rhys factors, $S_{n}=\Delta_{n}^{2} / 2$. The fluorescence band shape as a function of the frequency $\omega$ is determined by the imaginary part of the polarizability ${ }^{27,36}$

$$
\alpha(\omega)=\operatorname{Im}\left\{\mu^{2} \sum_{v_{1}} \cdots \sum_{v_{3 N-6}} \frac{\prod_{n=1}^{3 N-6}\left\langle 0 \mid v_{n}\right\rangle^{2}}{\Omega^{(0)}-\sum_{n=1}^{3 N-6} v_{n} \omega_{n}-\omega-i \Gamma}\right\}
$$

where $\mu$ is the electronic transition dipole moment between the excited and ground states, $\Omega^{(0)}$ is the associated $0-0$ transition energy, the $\omega_{n}$ 's are the vibrational frequencies, the $v_{n}$ 's are the number of quanta of the participating normal modes, and $\Gamma$ is an empirical parameter setting the spectral line widths. We choose the line width to be $0.01 \mathrm{eV}$ to allow fine resolution and analysis in terms of the contributing vibrational states.

Acknowledgment. We thank Prof. V. Y. Chernyak (WSU), Prof. G. Lanzani (Milano), Prof. A. Jorio (Belo Horizonte), Dr. A. P. Shreve (LANL), and Dr. S. K. Doorn (LANL) for useful discussions. The research at LANL is supported by Center for Integrated Nanotechnology (CINT), Center for Nonlinear Studies (CNLS), and the BES program of the U.S. Department of Energy. This support is gratefully acknowledged.

\section{References}

(1) Saito, R.; Dresselhaus, G.; Dresselhaus, M. S. Physical Properties of Carbon Nanotubes; Imperial College Press: London, 1998.

(2) Dresselhaus, M. S.; Dresselhaus, G.; Avouris, P. Carbon Nanotubes: Synthesis, Structure, Properties, and Applications; Springer: Berlin, 2001.

(3) Postma, H. W. C.; Teepen, T.; Yao, Z.; Grifoni, M.; Dekker, C Science 2001, 293, 76

(4) Misewich, J. A.; Martel, R.; Avouris, P.; Tsang, J. C.; Heinze, S.; Tersoff, J. Science 2003, 300, 783.

(5) Snow, E. S.; Perkins, F. K.; Houser, E. J.; Badescu, S. C.; Reinecke, T. L. Science 2005, 307, 1942.

(6) Zhang, M.; Fang, S. L.; Zakhidov, A. A.; Lee, S. B.; Aliev, A. E.; Williams, C. D.; Atkinson, K. R.; Baughman, R. H. Science 2005, 309, 1215.

(7) Rao, S. G.; Huang, L.; Setyawan, W.; Hong, S. H. Nature 2003 , 425, 36.

(8) Bachilo, S. M.; Strano, M. S.; Kittrell, C.; Hauge, R. H.; Smalley, R. E.; Weisman, R. B. Science 2002, 298, 2361.

(9) Ma, Y.-Z.; Valkunas, L.; Dexheimer, S. L.; Bachilo, S. M.; Fleming, G. R. Phys. Rev. Lett. 2005, 94, 157402.

(10) Korovyanko, O. J.; Sheng, C. X.; Vardeny, Z. V.; Dalton, A. B.; Baughman, R. H. Phys. Rev. Lett. 2004, 92, 017403.

(11) Kane, C. L.; Mele, E. J. Phys. Rev. Lett. 2004, 93, 197402.

(12) Ando, T. J. Phys. Soc. Jpn. 1997, 66, 1066

(13) Zhao H. B.; Mazumdar, S. Phys. Rev. Lett. 2004, 93, 157402.

(14) Perebeinos, V.; Tersoff, J.; Avouris, P. Phys. Rev. Lett. 2004, 92 257402.

(15) Chang, E.; Bussi, G.; Ruini, A.; Molinari, E. Phys. Rev. Lett. 2004, 92, 196401

(16) Spataru, C. D.; Ismail-Beigi, S. Benedict, L. X.; Louie, S. G. Phys Rev. Lett. 2004, 92, 774021.

(17) O’Connell, M. J.; Bachilo, S. M.; Huffman, C. B.; Moore, V. C.; Strano, M. S.; Haroz, E. H.; Rialon, K. L.; Boul, P. J.; Noon, W. H.; Kittrell, C.; Ma, J.; Hauge, R. H.; Weisman, R. B.; Smalley, R. E. Science 2002, 297, 593. 
(18) LeRoy, B. J.; Lemay, S. G.; Kong, J.; Dekker, C. Nature 2004, 432, 371.

(19) Chou, S. G.; Plentz, F.; Jiang, J.; Saito, R.; Nezich, D.; Ribeiro, H. B.; Jorio, A.; Pimenta, M. A.; Samsonidze, Ge. G.; Santos, A. P.; Zheng, M.; Onoa, G. B.; Semke, E. D.; Dresselhaus, G.; Dresselhaus, M. S. Phys. Rev. Lett. 2005, 94, 127402.

(20) Htoon, H.; O'Connell, M. J.; Cox, P. J.; Doorn, S. K.; Klimov, V. I. Phys. Rev. Lett. 2004, 93, 027401.

(21) Fantini, C.; Jorio, A.; Souza, M.; Strano, M. S.; Dresselhaus, M. S.; Pimenta, M. A. Phys. Rev. Lett. 2004, 93, 147406.

(22) Gambetta, A.; Manzoni, C.; Menna, E.; Meneghetti, M.; Cerullo, G.; Lanzani, G.; Tretiak, S.; Piryatinski, A.; Saxena, A.; Martin, R. L.; Bishop, A. R. Nat. Phys. 2006, 2, 515.

(23) Perebeinos, V.; Tersoff, J.; Avouris, P. Phys. Rev. Lett. 2005, 94, 027402 .

(24) Plentz, F.; Ribeiro, H. B.; Jorio, A.; Strano, M. S.; Pimenta, M. A Phys. Rev. Lett. 2005, 95, 247401.

(25) Habenicht, B. F.; Craig, C. F.; Prezhdo, O. V. Phys. Rev. Lett. 2006, 96, 187401.

(26) Heeger, A. J.; Kivelson, S.; Schrieffer, J. R.; Su, W. P. Rev. Mod. Phys. 1988, 60, 781.

(27) Tretiak, S.; Mukamel, S. Chem. Rev. 2002, $102,3171$.

(28) Batista, E. R.; Martin, R. L. J. Phys. Chem. A 2005, 109, 9856.

(29) Dexheimer, S. L.; VanPelt, A. D.; Brozik, J. A.; Swanson, B. I. Phys. Rev. Lett. 2000, 84, 4425

(30) Peierls, R. E. Quantum Theory of Solids; Clarendon: Oxford, 1955.
(31) Figge, M. T.; Mostovoy, M.; Knoester, J. Phys. Rev. Lett. 2001, 86, 4572.

(32) Luis, E. F.; Torres, F.; Roche, S. Phys. Rev. Lett. 2006, 97, 076804.

(33) Rafailov, P. M.; Maultzsch, J.; Thomsen, C.; Kataura, H. Phys. Rev. B 2005, 72, 045411 .

(34) Connetable, D.; Rignanese, G. M.; Charlier, J. C.; Blase, X. Phys. Rev. Lett. 2005, 94, 015503

(35) Bohnen, K. P.; Heid, R.; Liu, H. J.; Chan, C. T. Phys. Rev. Lett 2004, 93, 245501.

(36) Tretiak, S.; Saxena, A.; Martin, R. L.; Bishop, A. R. Phys. Rev. Lett. 2002, 89, 097402.

(37) Qiu, X. H.; Freitag, M.; Perebeinos, V.; Avouris, P. Nano Lett. 2005, $5,749$.

(38) Shreve, A. P.; Haroz, E. H.; Bachilo, S. M.; Weisman, R. B.; Tretiak, S.; Kilina, S.; Doorn, S. K. Determination of Exciton-Phonon Coupling Elements in Single-Walled Carbon Nanotubes by Raman Overtone Analysis, Phys. Rev. Lett. 2006, in press.

(39) Dewar, M. J. S.; Zoebisch, E. G.; Healy, E. F.; Stewart, J. J. P. J. Am. Chem. Soc. 1985, 107, 3902.

(40) Stewart, J. J. P. MOPAC 2002; Schrödinger Inc., Fujitsu Limited: Portland, OR, 2002.

(41) Zhou, Z. Y.; Steigerwald, M.; Hybertsen, M.; Brus, L.; Friesner, R. A. J. Am. Chem. Soc. 2004, 126, 3597.

NL0622000 